\title{
Autonomous Energy Harvesting Base Stations With Minimum Storage Requirements
}

\author{
Panagiotis D. Diamantoulakis, Student Member, IEEE, Koralia N. Pappi, Member, IEEE, \\ George K. Karagiannidis, Fellow, IEEE, and H. Vincent Poor, Fellow, IEEE
}

\begin{abstract}
In this work, optimal energy and resource allocation for the downlink of an autonomous energy-harvesting base station is investigated. In particular, the joint maximization of the users' utilities and the base station's revenue is considered, while these hierarchical decision problems are matched to the framework of a generalized Stackelberg game. Also, an efficient iterative method is proposed that enables all the players to reach the variational equilibrium, i.e., the optimal solution of the game. Simulation results validate the effectiveness of the proposed method.
\end{abstract}

Index Terms-Energy harvesting, base stations, energy autonomous systems, resource allocation, Stackelberg game.

\section{INTRODUCTION}

$\mathbf{T}$ HE deployment of energy-harvesting base stations (EHBSs) is a promising solution for the reduction of operating expenses in wireless communication networks. EHBSs use the harvested power from the wind, solar radiation, etc. to satisfy a part of their energy demand [1], [2]. With advances in the speed of wireless backhaul that eliminate the need for wired connection, autonomous energy harvesting base stations (AEHBSs) are of particular interest, especially for small cells [3] or for use as relay stations [4]. In [5], a novel method is proposed that maximizes the energy efficiency in systems with hybrid EHBSs (HEHBSs), using both the electricity grid and energy harvesting as power sources. In HEHBSs, user fairness can be achieved by setting a minimum rate requirement for each user as a minimum level of satisfaction. However, in an AEHBS that is solely powered by energy harvesting, this is not always possible since the BS may not have enough energy [3]. The probability of meeting this requirement can be increased by employing larger harvesting and storage equipment; however this also substantially increases capital expenditures.

In this work, we investigate user fairness in AEHBSs with minimum energy storage requirements. A novel scheme is proposed, in which the users pay per amount of utilized resources and energy used for transmission. We define a utility function for each user, which reflects the user experience and satisfaction, as an alternative fairness requirement. In this framework,

Manuscript received January 27, 2015; revised February 9, 2015; accepted February 10, 2015. Date of publication February 24, 2015; date of current version June 18,2015 . This work was supported by the NPRP grant \# NPRP 6-1326-2-532 from the Qatar National Research Fund (a member of Qatar Foundation). The statements made herein are solely the responsibility of the authors. The associate editor coordinating the review of this paper and approving it for publication was D. Niyato.

P. D. Diamantoulakis, K. N. Pappi, and G. K. Karagiannidis are with the Department of Electrical and Computer Engineering, Aristotle University of Thessaloniki, Thessaloniki 54 124, Greece (e-mail: padiaman@auth.gr; kpappi@auth.gr; geokarag@auth.gr).

H. V. Poor is with the Department of Electrical Engineering, Princeton University, Princeton, NJ 08544 USA (e-mail: poor@ princeton.edu).

Digital Object Identifier 10.1109/LWC.2015.2406711 the users' objective is to maximize their levels of satisfaction, while the base station (BS) aims to maximize its revenue, accommodating all the users over the finite available spectrum. To find the optimal solution of this hierarchical decision problem, we use game theoretic tools, and specifically Stackelberg games [6]. Also, to minimize the storage requirements we consider that in a specific time frame, the BS can only use the energy harvested in the previous time frame. Results show that previously proposed solutions, such as maximizing the total capacity for a specific amount of energy, are not fair solutions and do not optimize the users' experience in an AEHBS.

\section{System Model}

We consider a wireless network consisting of $N$ users and one autonomous energy harvesting base station. The communication is divided into time frames of duration $T$, while the total bandwidth is denoted by $B$. The communication with each user occupies a distinct fraction of time and frequency, so that the signals of different users do not interfere. At the time instant $t$ within a frame $k$, the BS transmits to the $n^{\text {th }}$ user with power $P_{n}(t)$, using the bandwidth fraction $w_{n}(t)$. For the link between the BS and the $n^{\text {th }}$ user, $g_{n}(t)$ and $h_{n}(t)$ denote the path loss/shadowing coefficient and the small scale fading coefficient, respectively. The time fraction allocated to the $n^{\text {th }}$ user is denoted by $\tau_{n}$.

We assume that the energy consumed by the BS is limited by the amount of harvested energy, i.e., in time frame $k$, the BS can only use the energy that was harvested during the time frame $k-1$, a strategy that creates very low storage requirements. For notational simplicity, we further assume that the BS consumes energy only for transmission, which is denoted by $E$. If $E_{n}$ is the fraction of the energy dedicated for the $n^{\text {th }}$ user, it holds that $\sum_{n=1}^{N} E_{n} \leq E$.

The channel capacity between the BS and the $n^{\text {th }}$ user in a frame $k$ is given by the effective rate (bits per time frame) that each user can achieve during the frame $T$, which, assuming additive white Gaussian noise, is given by [5]

$$
\mathcal{C}_{n}=\int_{0}^{T} s_{n}(t) w_{n}(t) \log _{2}\left(1+\frac{P_{n}(t) G_{n}(t)}{w_{n}(t)}\right) \mathrm{d} t .
$$

In (1), $s_{n}(t) \in\{0,1\}$ denotes the time allocation with $\int_{0}^{T} s_{n}(t) \mathrm{d} t=\tau_{n}$, and $G_{n}(t)=\frac{g_{n}(t)\left|h_{n}(t)\right|^{2}}{N_{0}}$, where $N_{0}$ is the noise power spectral density. To simplify theoretical studies we assume that the coherence time and bandwidth of the channel are larger than $T$ and $B$ respectively (see [7] and references therein). We also assume that the power transmitted towards each user is constant, and thus $P_{n}=\frac{E_{n}}{\tau_{n}}$. Therefore all values are frequency flat and constant within each frame $k$, that is, independent of $t$. The analysis focuses on a specific time frame, 
and $t$ is therefore omitted hereafter. If the time and bandwidth resources ${ }^{1}$ for each user are denoted by $q_{n}$, where $q_{n}=\tau_{n} w_{n}$, then the channel capacity can be simplified to

$$
\mathcal{C}_{n}=q_{n} \log _{2}\left(1+\frac{E_{n}}{q_{n}} G_{n}\right) .
$$

The sum of the available resources must satisfy the condition $\sum_{n=1}^{N} q_{n} \leq Q$, where $Q=T B$. Hereafter, $\mathbf{E}=\left\{E_{1}, \ldots, E_{N}\right\}$ and $\mathbf{q}=\left\{q_{1}, \ldots, q_{N}\right\}$ denote the sets of values of the energy and resource allocation among users within a frame $k$, respectively.

\section{Problem Formulation}

We assume that the BS charges the users according to the resources and the transmitted energy they use during a time frame $k$. Both the users and the BS want to maximize their utility functions, i.e., their levels of satisfaction and their revenue, respectively.

\section{A. Objective of the $B S$}

The BS can control the price $p_{1}$ per utilized energy unit (e.g., $\$ / \mathrm{J}$ ) and $p_{2}$ per unit of resources (e.g., \$) to maximize its utility function. The utility function $R$ of the BS captures the revenue (e.g., \$) that the operator can receive by selling its resources and energy, and it is given by

$$
R=p_{1} \sum_{n=1}^{N} E_{n}+p_{2} \sum_{n=1}^{N} q_{n} .
$$

\section{B. Objective of the Users}

The users respond to the prices by demanding a certain amount of resources and energy, to maximize their utilities, $U_{n}$. For each user $n \in \mathcal{N}$, where $\mathcal{N}$ is the set of all the users, the utility function corresponds to a level of satisfaction that a user obtains [8]. The following assumptions are made for the utility functions of the users: (i) Each is affected by a satisfaction parameter $a_{n}$ of each user. This parameter is different according to the appliance of the user or the service it requires, i.e., a smart phone or a tablet that is usually online will have higher demands for rate than a simple cell phone or a smart meter. Generally, a higher $a_{n}$ implies a higher level of satisfaction. Thus, $U_{n}$ must be an increasing function with respect to $a_{n}$. (ii) Each is a decreasing function of the prices $p_{1}$ and $p_{2}$, since higher prices lead to a lower level of satisfaction. (iii) Each reflects the corresponding user's desire for higher capacity as well as the saturation of its satisfaction level as higher capacity is achieved [9]. This is ensured when, apart from the payment $\left(p_{1} E_{n}+p_{2} q_{n}\right), U_{n}$ includes a term that is an increasing and concave function of $C_{n}$.

In this work, we consider the following utility function for the users:

$$
U_{n}=a_{n} \log _{2}\left(1+\mathcal{C}_{n}\right)-p_{1} E_{n}-p_{2} q_{n}, \quad a_{n}, p_{1}, p_{2}>0 .
$$

In the above, the weight $a_{n}$, multiplied by the term quantifying the rate, captures the satisfaction acquired by the quality of service, while the subtraction-or equivalently, a negative unitary weight multiplied by the term concerning the payment-

\footnotetext{
${ }^{1}$ The term "resource" herein refers to bandwidth/time and not energy.
}

captures the dissatisfaction induced by the users' charges. Therefore, $U_{n}$ is measured in units of satisfaction. Thus, for a fixed set of prices $p_{1}$ and $p_{2}$, the objective of any user $n$ is

$$
\begin{aligned}
& \max _{q_{n}, E_{n}} U_{n}\left(p_{1}, p_{2}, E_{n}, \mathbf{E}_{-n}, q_{n}, \mathbf{q}_{-n}\right) \\
& \text { s.t. } C_{1}: \sum_{n=1}^{N} E_{n} \leq E, C_{2}: \sum_{n=1}^{N} q_{n} \leq Q,
\end{aligned}
$$

where $\mathbf{E}_{-n}$ and $\mathbf{q}_{-n}$ denote the sets $\mathbf{E}$ and $\mathbf{q}$, excluding $E_{n}$ and $q_{n}$, respectively.

\section{Stackelberg Game}

The hierarchical decision problem presented in Section III can be effectively solved using a Stackelberg game. In this hierarchical game, the followers decide in response to the decision taken by the leader. In this context, at each decision point the users (the followers) know the prices set by the BS (the leader), while the BS has knowledge of the users' strategies, i.e., the demanded energies and resources. Consequently, the $N$ users form a lower level game. When this lower level game achieves an equilibrium, the equilibrium together with the operator (BS) form the higher level game. Then, the equilibrium of the higher level is called the Stackelberg Equilibrium (SE) and it is the solution of the game [6]. This game is defined in its strategic form as follows:

$$
\Gamma=\left\{(\mathcal{N} \cup B S),\left\{E_{n}, q_{n}\right\}_{n \in \mathcal{N}},\left\{U_{n}\right\}_{n \in \mathcal{N}}, R, p_{1}, p_{2}\right\} .
$$

\section{A. Generalized Stackelberg Equilibrium}

In (5), the amount of resources and energy requested by each user depend not only on its own strategy (resources and energy demand) but also on the strategies of the other users, since they share the same constraints. Thus, the noncooperative game among the users represents a generalized Nash Equilibrium problem (GNEP), the solution of which is the generalized Nash Equilibrium (GNE) [10], [11]. Moreover, since the objective of the BS is to maximize its utility, $R\left(p_{1}, p_{2}, \mathbf{E}^{*}, \mathbf{q}^{*}\right)$, taking into account the GNE of the users' game, the formulated game $\Gamma$ represents a generalized Stackelberg game, the solution of which is the generalized SE (GSE) [12]. The set of strategies $\left(p_{1}^{*}, p_{2}^{*}, \mathbf{E}^{*}, \mathbf{q}^{*}\right)$ that constitutes the GSE is given by solving the following inequalities, where $(\cdot)^{*}$ denotes a solution value:

$$
\begin{aligned}
& U_{n}\left(p_{1}^{*}, p_{2}^{*}, E_{n}^{*}, \mathbf{E}_{-n}^{*}, q_{n}^{*}, \mathbf{q}_{-n}^{*}\right) \\
& \quad \geq U_{n}\left(p_{1}^{*}, p_{2}^{*}, E_{n}, \mathbf{E}_{-n}^{*}, q_{n}, \mathbf{q}_{-n}^{*}\right), \quad \forall n \in \mathcal{N}, \\
& R\left(p_{1}^{*}, p_{2}^{*}, \mathbf{q}^{*}, \mathbf{E}^{*}\right) \geq R\left(p_{1}, p_{2}, \mathbf{q}^{*}, \mathbf{E}^{*}\right), C_{1}, C_{2} .
\end{aligned}
$$

\section{B. Existence and Uniqueness of the GNE}

A GNEP can be solved by using a variational inequality (VI) problem reformulation. The GNEP might have multiple or even infinitely many solutions; however each solution of the GNEP is not always a solution of the VI [13]. A solution of the GNEP which is also a solution of the VI is called a variational equilibrium (VE) and is considered to be the most socially stable GNE [11]. 
Since $F=\left(\nabla_{E_{1}} U_{1}, \nabla_{q_{1}} U_{1}, \ldots, \nabla_{E_{N}} U_{N}, \nabla_{q_{N}} U_{N}\right)$ is the gradient of a scalar function, i.e., $F=\nabla f$ with $f=\sum_{n=1}^{N} U_{n}$, the variational solutions are the solutions of the following optimization problem [13]:

$$
\begin{aligned}
\underset{\mathbf{q}, \mathbf{E}}{\max } & f(\mathbf{q}, \mathbf{E}) \\
\text { s.t. } & C_{1}, C_{2} .
\end{aligned}
$$

Then, we present the following theorem.

Theorem 1: For fixed prices $p_{1}$ and $p_{2}$, a social VE exists, which is unique.

Proof: To accommodate the proof of the existence and uniqueness of a VE, we assume that $q_{n} \in[\epsilon, Q]$ and $E_{n} \in$ $[\varepsilon, E]$, where $\epsilon, \varepsilon \rightarrow 0^{+}$. With the aid of the above assumption, it can be shown that the Hessian matrix of $U_{n}$ has negative eigenvalues, so $U_{n}$ is jointly strictly concave with respect to the optimization variables $q_{n}$ and $E_{n}$. Also, the sum over $n$ preserves the concavity of the objective function, $f$, in (8), while the constraints $C_{1}$ and $C_{2}$ are linear. Thus, (8) is strictly concave, which means that it has a unique solution and so does the equivalent VI.

\section{Solution of the Game}

1) Solving the VI: Using the Lagrange multiplier method, the Lagrangian for the optimization problem (8) is

$$
\mathcal{L}=f(\mathbf{q}, \mathbf{E})-\lambda_{1}\left(\sum_{n=1}^{N} E_{n}-E\right)-\lambda_{2}\left(\sum_{n=1}^{N} q_{n}-Q\right)
$$

where $\lambda_{1}, \lambda_{2} \geq 0$ are the Lagrange multipliers related to $C_{1}, C_{2}$, respectively. The values $\mathbf{q}^{*}, \mathbf{E}^{*}, \lambda_{1}^{*}$ and $\lambda_{2}^{*}$ that satisfy the Karush-Kuhn-Tucker (KKT) conditions can be obtained iteratively as follows: in Layer 1 the users simultaneously choose their demands for a fixed set of $\lambda_{1}$ and $\lambda_{2}$, the values of which are updated in Layer 2 by the BS.

Layer 1 (Solved by the users): From the KKT conditions it must hold that $\frac{\partial \mathcal{L}}{d E_{n}}=0$ and $\frac{\partial \mathcal{L}}{d q_{n}}=0$. So, with direct calculations we obtain the optimal values of $E_{n}$ and $q_{n}$ in a distributed way, since they can be calculated in parallel by each user according to

$$
\begin{aligned}
& E_{n}^{*}=\left[\frac{\left(e^{D_{n}}-1\right)\left(\frac{a_{n} G_{n} e^{-D_{n}}}{l_{1}}-(\ln (2))^{2}\right)}{D_{n} G_{n} \ln (2)}\right]_{\varepsilon}, \\
& q_{n}^{*}=\left[\frac{\frac{a_{n} G_{n} e^{-D_{n}}}{l_{1}}-(\ln (2))^{2}}{D_{n} \ln (2)}\right]_{\epsilon},
\end{aligned}
$$

where $[.]_{\xi}=\max (., \xi)$, and $D_{n}$ is given by

$$
D_{n}=1+W\left(\frac{-l_{1}+l_{2} G_{n}}{e l_{1}}\right)
$$

where $l_{1}=\lambda_{1}+p_{1}$ and $l_{2}=\lambda_{2}+p_{2}$. Moreover, $W(x)$ denotes the Lambert $\mathrm{W}$ function, also called the omega function or product logarithm, which gives the principal solution for $W(x)$ in $x=W(x) e^{W(x)}$. Note that $W(x)$ is a built-in function in most well-known mathematical software packages.
Layer 2 (Solved by the BS): Using the dual-domain and subgradient methods, $\lambda_{1}$ and $\lambda_{2}$ can be obtained by

$$
\begin{aligned}
& \lambda_{1}(\nu+1)=\left[\lambda_{1}(\nu)-\zeta_{1}(\nu)\left(E-\sum_{n=1}^{n} E_{n}(\nu)\right)\right]_{0} \\
& \lambda_{2}(\nu+1)=\left[\lambda_{2}(\nu)-\zeta_{2}(\nu)\left(Q-\sum_{n=1}^{n} q_{n}(\nu)\right)\right]_{0}
\end{aligned}
$$

where $\zeta_{j}(\nu), j \in\{1,2\}$ are positive step sizes, chosen to satisfy the diminishing step size rules and $\nu>0$ is the iteration index. Since the optimization problem (8) is concave, it is guaranteed that the iteration between the two layers converges to the optimal solution of (8) [14], [15].

It is remarkable that by adopting the proposed method, each user can separately calculate its own optimized demand in each iteration, and thus the users can reach the VE with no communication among themselves. In contrast, they are only required to communicate with the BS, a fact that substantially reduces the overhead. These benefits fully justify the selection of the proposed distributed solution method.

2) Optimizing the Prices: From the KKT conditions it holds that

$$
\frac{\partial U_{n}}{\partial E_{n}}-\lambda_{1}^{*}=0, \quad \frac{\partial U_{n}}{\partial q_{n}}-\lambda_{2}^{*}=0 .
$$

Since, $\lambda_{1}^{*} \geq 0$ and $\lambda_{2}^{*} \geq 0$, the prices $p_{1}$ and $p_{2}$ must satisfy the following inequalities:

$$
\begin{aligned}
& p_{1} \leq \frac{a_{n} G_{n} q_{n}^{*}}{\left(G_{n} E_{n}^{*}+q_{n}^{*}\right)\left(\ln (2)+q_{n}^{*} \ln \left(1+\frac{G_{n} E_{n}^{*}}{q_{n}^{*}}\right)\right) \ln (2)} \\
& p_{2} \leq \frac{a_{n}\left(-G_{n} E_{n}^{*}+\left(G_{n} E_{n}^{*}+q_{n}^{*}\right) \ln \left(1+\frac{G_{n} E_{n}^{*}}{q_{n}^{*}}\right)\right)}{\left(G_{n} E_{n}^{*}+q_{n}^{*}\right)\left(\ln (2)+q_{n}^{*} \ln \left(1+\frac{G_{n} E_{n}^{*}}{q_{n}^{*}}\right)\right) \ln (2)} .
\end{aligned}
$$

The revenue maximizing prices $p_{1}^{*}$ and $p_{2}^{*}$ are given by (16) and (17), respectively, when these hold with equality.

\section{Results AND Discussion}

For the simulation results, we consider the case $g_{n}=1$, $Q=1$ and $a_{n}$ chosen randomly and uniformly in the range of $[1,2]$. All statistical results are averaged over random values of $h_{n}$ and $a_{n}$, where $h_{n} \sim \mathcal{C N}(0,1)$. All the provided results take into account the effects of the number of users and the different amount of harvested energy. To do so, we plot the desired metric versus the harvested energy, $\frac{E}{Q N_{0}}$, for three cases for the number of users, i.e., $N=5,10,20$.

In Fig. 1 the average prices $p_{1}^{*}$ and $p_{2}^{*}$ are illustrated. It is seen that the optimal prices increase with the number of users. This implies that the demand is increasing, while the available resources and energy are limited. On the other hand, when the harvested energy increases, the price per unit of energy reduces (e.g., $p_{1}^{*}=0.00109$ for $N=5$ and $\frac{E}{Q N_{0}}=30 \mathrm{~dB}$ ), since the BS has more energy to cover the demand. In contrast to $p_{1}^{*}$, increasing the harvested energy leads to an increase in the optimum price $p_{2}^{*}$. This is a notable observation, because it indicates that the more the transmitted power increases, the smaller the increase of the users' utilities is, and thus, instead of demanding energy, the demand for the other resources increases. 


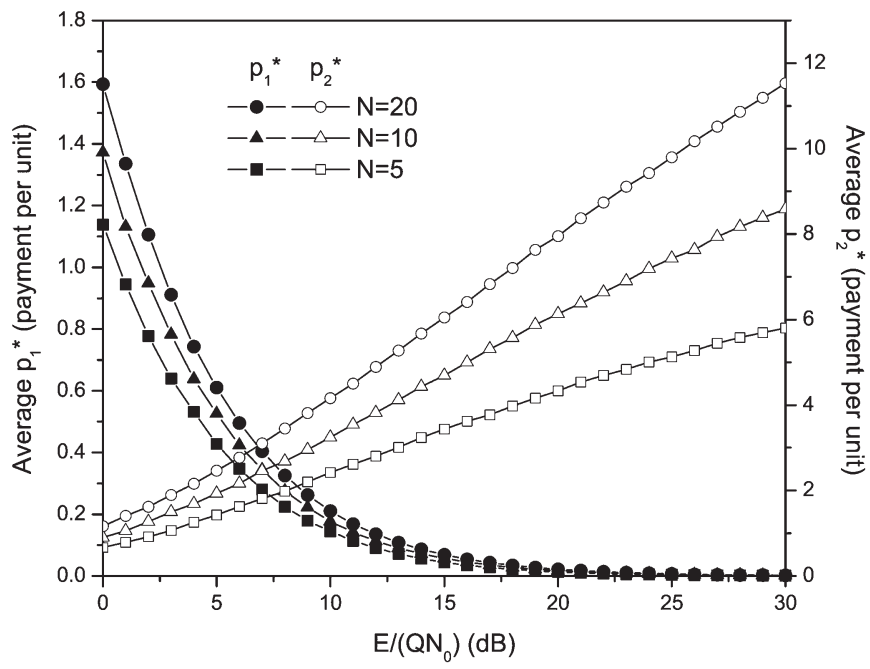

Fig. 1. Average prices $p_{1}^{*}$ and $p_{2}^{*}$ per unit of energy and resources, respectively.

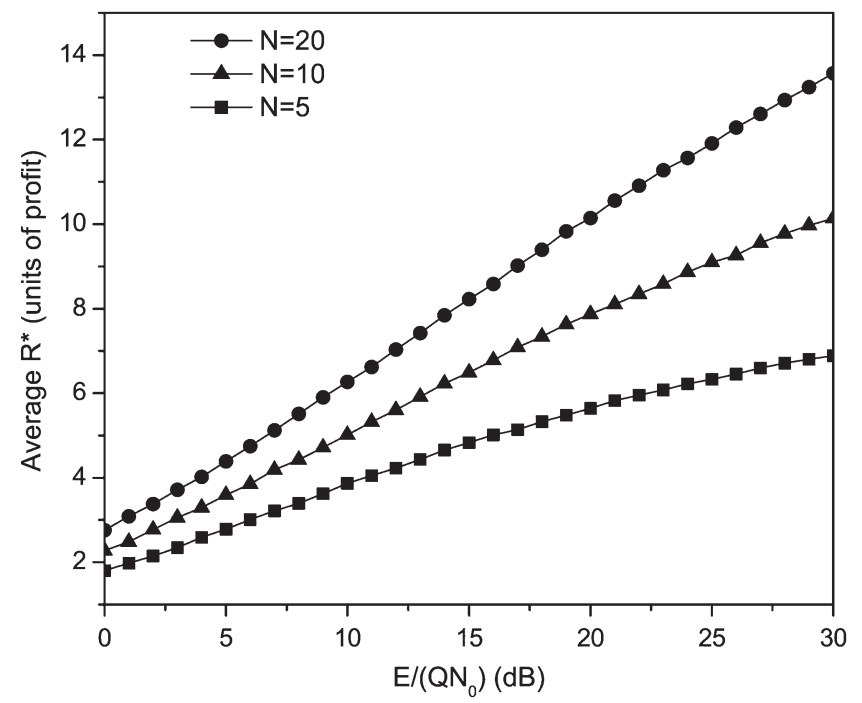

Fig. 2. Average revenue $R^{*}$ of the base station.

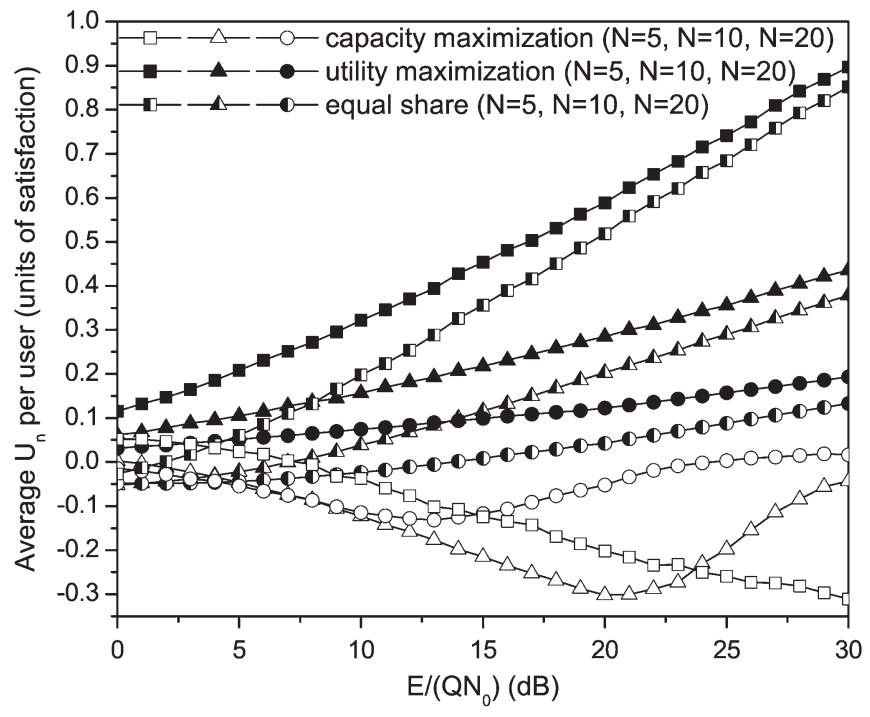

Fig. 3. Comparison of the average utility per user.

Figs. 2 and 3 show the average BS revenue and the average utility per user, respectively. It can be observed that when the proposed method (utility maximization) is applied, by increasing the harvested energy, both $R_{n}^{*}$ and $U_{n}^{*}$ also increase, i.e., when the BS harvests more energy it increases its revenue, because of the increase of prices in this case, while the users are more satisfied, since their spectral efficiency increases. In Fig. 3, in conjunction with the utility maximization, two other energy and resource allocation strategies are illustrated: maximization of the total capacity of the network (capacity maximization), and equal power and resource sharing among the users. One can observe that the utility maximization method outperforms both schemes, reflecting the fairness that is achieved among users and underscoring the value of the gametheoretic approach followed in this work.

\section{CONCLUSION}

In this paper, we have used a generalized Stackelberg game to investigate the optimal energy and resource allocation problem of an autonomous energy harvesting base station. We have proved existence and uniqueness of a variational equilibrium for this game. We have also proposed an efficient distributed algorithm, which can be adopted by the players to maximize their utility functions. The simulation results have revealed that by applying the proposed method, the users maximize their levels of satisfaction while the BS maximizes its revenue for all values of harvested energy and for an arbitrary number of users.

\section{REFERENCES}

[1] C. Wang and M. H. Nehrir, "Power management of a stand-alone wind/ photovoltaic/fuel cell energy system," IEEE Trans. Energy Convers., vol. 23, no. 3, pp. 957-967, Sep. 2008.

[2] P. D. Diamantoulakis, A. Ghassemi, and G. K. Karagiannidis, "Smart hybrid power system for base transceiver stations with real-time energy management," in Proc. IEEE GLOBECOM, Dec. 2013, pp. 2773-2778.

[3] H. S. Dhillon, Y. Li, P. Nuggehalli, Z. Pi, and J. G. Andrews, "Fundamentals of heterogeneous cellular networks with energy harvesting," IEEE Trans. Wireless Commun., vol. 13, no. 5, pp. 2782-2797, May 2014.

[4] Y. Song, M. Zhao, W. Zhou, and H. Han, "Throughput-optimal user association in energy harvesting relay-assisted cellular networks," in Proc. 6th Int. Conf. WCSP, Oct. 2014.

[5] D. W. K. Ng, E. S. Lo, and R. Schober, "Energy-efficient resource allocation in OFDMA systems with hybrid energy harvesting base station," IEEE Trans. Wireless Commun., vol. 12, no. 7, pp. 3412-3427, Jul. 2013.

[6] T. Basar and G. J. Olsder, Dynamic Noncooperative Game Theory. Philadelphia, PA, USA: SIAM, 1999.

[7] C. Xu, M. Sheng, C. Yang, X. Wang, and L. Wang, "Pricing-based multiresource allocation in OFDMA cognitive radio networks: An energy efficiency perspective," IEEE Trans. Veh. Technol., vol. 63, no. 5, pp. 2336-2348, Jun. 2014.

[8] F. Meshkati, H. V. Poor, and S. C. Schwartz, "Energy-efficient resource allocation in wireless networks," IEEE Signal Process. Mag., vol. 24, no. 3, pp. 58-68, May 2007.

[9] V. Gajic, J. Huang, and B. Rimoldi, "Competition of wireless providers for atomic users," IEEE/ACM Trans. Netw., vol. 22, no. 2, pp. 512-525, Apr. 2014.

[10] F. Facchinei and C. Kanzow, "Generalized nash equilibrium problems," 4OR, vol. 5, no. 3, pp. 173-210, Sep. 2007.

[11] W. Tushar, W. Saad, H. V. Poor, and D. B. Smith, "Economics of electric vehicle charging: A game theoretic approach," IEEE Trans. Smart Grid, vol. 3, no. 4, pp. 1767-1778, Dec. 2012.

[12] G. Leitmann, "On generalized stackelberg strategies," J. Optim. Theory Appl., vol. 26, no. 4, pp. 637-643, Dec. 1978.

[13] G. Scutari, D. P. Palomar, F. Facchinei, and J.-S. Pang, "Convex optimization, game theory, variational inequality theory," IEEE Signal Process. Mag., vol. 27, no. 3, pp. 35-49, May 2010.

[14] S. Boyd and L. Vandenberghe, Convex Optimization. Cambridge, U.K.: Cambridge Univ. Press, 2009.

[15] S. Boyd, L. Xiao, and A. Mutapcic, Subgradient Methods, Lecture Notes of EE392o. Stanford, CA, USA: Stanford Univ. Press, 2003-2004. 